\title{
Clinical features and prognosis analysis of Hodgkin's lymphoma: A multicenter retrospective study over a decade of patients in China
}

Running title: Clinical analysis and new risk model of $\mathrm{HL}$

Wen-Yan YU ${ }^{1}$, Mei Geng ${ }^{2}$, Jie Hao ${ }^{3}$, Mei Chen ${ }^{4}$, Su-Jiang Zhang ${ }^{5}$, Jin Wang ${ }^{1}$, Jian-Qing Mi ${ }^{1}$

${ }^{1}$ Shanghai Institute of Hematology, Rui Jin Hospital Affiliated to Shanghai Jiao Tong University School of Medicine, Shanghai, China;

${ }^{2}$ Department of Oncology, Rui Jin Hospital Affiliated to Shanghai Jiao Tong University School of Medicine, Shanghai, China;

${ }^{3}$ Department of Hematology, Shanghai North Station Hospital, Shanghai, China;

${ }^{4}$ Department of Hematology, Shanghai Yang Pu Central Hospital Affiliated to Shanghai Tong Ji University, Shanghai, China;

${ }^{5}$ Department of Hematology, Rui Jin North Medical Center Affiliated to Shanghai Jiao Tong University School of Medicine, Shanghai, China;

Correspondence Jian-Qing MI, M.D. \& Ph.D, State Key Laboratory for Medical Genomics and Department of Hematology, Shanghai Institute of Hematology, Collaborative Innovation Center of Systems Biomedicine, Pôle Sino-Français des Sciences du Vivant et Genomique, Rui Jin Hospital, Shanghai Jiao Tong University School of Medicine, 197 Rui Jin Er Road, Shanghai 200025, China. Tel: 0086-21-33562658; Fax: 0086-21-64743206; E-mails: jianqingmi@ shsmu.edu.cn

Article Type: Original Article 


\title{
Clinical features and prognosis analysis of Hodgkin's lymphoma:
}

\section{A multicenter retrospective study over a decade of patients in China}

\author{
Abstract \\ Objective: Little information of Hodgkin's lymphoma (HL) is available in Chinese patients. We \\ analyzed their clinical features, outcome and prognostic factors aiming to establish a new risk model \\ for better risk-adapted therapeutic strategy.
}

Patients and methods: Patients with newly diagnostic HL at 4 medical centers from January 2000 to August 2014 were recruited.

Results: 150 patients were reviewed. Median age was 30y (range 15-91y). $73.65 \%$ of patients achieved complete remission (CR) at completion of initial therapy. The 5-year event-free survival (EFS) of the entire cohort was $61.1 \%$; the overall survival was $84.7 \%$; and the disease-free survival was $78.8 \%$. B symptoms; extranodal involvement and IPS $\geqslant 3$ remained as independent prognostic factors on EFS. Patients who failed to reach CR on interim PET/CT or CT had a significantly worse outcome than those who did. A new risk model incorporating traditional risk factors and interim response stratified patients into 3 classes, with 5-y EFS of 100\%, 83.1\% and 33.1\%, respectively $(\mathrm{P}<0.0001)$.

Conclusions: General clinical features were comparable to western patients, whereas therapeutic outcomes were slightly inferior. The novel risk assessment model showed potential as a more powerful prognostic tool by identifying 3 subsets of patients with significantly distinct outcomes, which warrants further validations.

\section{Keywords}

Hodgkin's lymphoma, clinical feature, treatment outcome, prognostic factors, risk model

\section{Introduction}

Over the last four decades, advances in radiotherapy and the addition of combination chemotherapy have significantly increased the cure rate of patients with Hodgkin's lymphoma (HL). More than $80 \%$ of newly diagnosed patients younger than $60 \mathrm{y}$ are likely to be cured ${ }^{[1-2]}$. Primary ABVD (Doxorubicin/Bleomycin/Vinblastine/Dacarbazine) combination chemotherapy, followed by involved-field irradiation (IF-RT), is regarded as the standard treatment for patients with early-stage HL. In addition, 6-8 cycles of ABVD with or without radiotherapy (RT) is recognized as the standard care for patients with advanced-stage HL with a 10 -year OS $>50 \%{ }^{[4]}$. More intensive combinations, such as BEACOPP (Bleomycin/ Etoposide/ Doxorubicin/ Cyclophosphamide/ Vincristine/ Procarbazine/ Prednisone) and Stanford V (Doxorubicin/ Vinblastine/ Mechlorethamine/ Vincristine/ Bleomycin/ Etoposide/ Prednisone), have been developed in the last decade to improve outcome, particularly for patients with poor prognosis. Regardless, in routine clinical practice, due to treatment-related toxicity or insufficient evaluation by large scale studies, these combinations have not fully replaced ABVD ${ }^{[5,6]}$. Till now, ABVD is still the stand approach for HL.

Current treatment for HL exhibits a tendency toward risk-adapted initial therapy. The definition of prognostic factors and risk groups remains inconclusive. Numerous clinical, histopathological and laboratory parameters have individually been suggested to be of prognostic value. Related factors are listed as following: bulky mediastinal mass; age; erythrocyte sedimentation rate（ESR）; B symptoms; 
extranodal site; nodal areas; histological type, etc. In addition, International Prognostic Score (IPS) proposed by Hasenclever D et al. ${ }^{[3]}$, remained widely used worldwide, including 7 adverse factors (age $\geq 45 \mathrm{y}$, male sex, stage IV, serum albumin $<4 \mathrm{~g} / \mathrm{dl}$, hemoglobin $<10.5 \mathrm{~g} / \mathrm{dl}$, white blood cell count $\geq 15 \times 10^{9} / \mathrm{L}$, and lymphocytes count $<0.6 \times 10^{9} / \mathrm{L}$ or less than $8 \%$ ). With the rapid development of imaging technology, early imaging evaluation has been proposed as a useful prognostic tool integrated in response-adapted therapy, such as interim 18F-fluorodeoxyglucose positron emission tomography with computerized tomography (PET/CT) after 2-4 cycles of chemotherapy ${ }^{[7,8]}$. Several researchers even suggested that traditional prognostic factors gradually lost their initial predictive power with the development of imaging technology. Therefore, more sensitive and specific risk stratification tool need to be explored to identify the high-risk group of patients.

Generally, the prognosis of tumors depends on several factors: disease-related factors, host-related factors, and treatment-related factors. To integrate these three parts, we tried to establish a simple and practical risk model, which combines certain traditional risk factors with interim imaging evaluation. Our goal is to achieve a higher prognostic assessment value. In addition, because of the low incidence of HL in an Asian population, reports on clinical features and treatment outcome among Asian patients are limited. In our study, with a follow-up of over a decade, we analyzed the characteristics, therapeutic outcome and prognostic factors of Chinese patients with HL. Our report is expected to provide supplementary information to related global studies, as well as be a reference for other Asian working groups.

\section{Materials and Methods}

\section{Patients}

We conducted a multicenter retrospective analysis of 150 patients consecutively diagnosed and treated with HL in 4 medical centers between January 2000 and August 2014. A tissue biopsy was performed in all patients for diagnostic purpose, all cases were pathologically confirmed by pathologists and classified in accordance with the 2008 WHO classification of hematological malignancies ${ }^{[11]}$. Patients were followed up using documentations gathered during hospitalization or by telephone and mail. The last follow-up was conducted in August 2015, and the median follow-up duration was 48.5 (4-133) months, including 20 cases (13.3\%) of incomplete data or loss of follow-up (in our study, data of patients lost to follow up were regarded as censored data).

\section{Stages and routine measures}

All patients were clinically staged in accordance with the Cotswold modifications of the Ann Arbor criteria ${ }^{[9]}$. Staging procedures included medical history and physical examination; chest radiography; computed tomography (CT) of the neck, chest, abdomen, and pelvis or PET-CT; and bone marrow biopsy. Bulky mediastinal disease was defined as the presence of mediastinal mass with a maximal width of more than one third of the intrathoracic maximal diameter on a standing posteroanterior chest X-ray. Clinical parameters included sex; age; histological subtype; initial symptoms at diagnosis; B-symptoms; involved anatomic sites and extranodal disease. In laboratory examinations, hemoglobin, white blood cell (WBC) counts and differential, erythrocyte sedimentation rate (ESR), serum albumin (ALB), serum $\beta 2$-microglobulin ( $\beta 2-\mathrm{MG})$, serum alkaline phosphatase (AKP), and serum lactate dehydrogenase (LDH) levels were measured by standard assays. In our study, the biochemical abnormalities mentioned below were defined as the presence of any of the following: 
LDH $>1 x$ Normal $\quad(192 \mathrm{U} / \mathrm{L}), \quad \beta 2-\mathrm{MG}>1 \mathrm{xNormal} \quad(1310 \mathrm{U} / \mathrm{L}), \quad$ AKP $>1 \mathrm{xNormal} \quad(121 \mathrm{U} / \mathrm{L}) \quad$ o ALB $<1 \mathrm{xNormal}(3.5 \mathrm{~g} / \mathrm{dl})$. The IPS was determined as described previously ${ }^{[3]}$.

\section{Treatment}

All newly diagnosed patients were initially treated with chemotherapy. Patients with stage I-II HL received 4-6 cycles of an anthracycline-based chemotherapy, whereas those with stage III-IV HL received 6-8 cycles of chemotherapy. In principle, all patients should be evaluated by CT or PET/CT for interim response after 2-4 cycles and be assessed for final response at the end of initial treatment (chemotherapy and radiotherapy).Starting within 1 month after the end of chemotherapy, patients with a complete response or partial response were suggested to receive 20-36Gy IFRT to nodal sites of initial bulky mediastinal disease, areas of residual disease, or both. Salvage treatment was given for those with refractory diseases, progressive disease or relapse during treatment or follow-up. High-dose therapy followed by autologous stem cell transplant was administered whenever possible. The majority of the 150 patients received ABVD regimen. Besides, three additional regimen including MOPP, COPP/ABVD and EACOP/ABVD had also been applied on another 4 patients. The modalities for salvage treatment included several regimens, such as ICE, GDP, IGEV, and ESHAP.

\section{Criteria for response and end points}

The primary end point is event-free survival (EFS) at $5 \mathrm{y}$ after diagnosis. It is defined for all patients and measured from the start of treatment until treatment failure, relapse from CR, progression or death from any cause. The treatment failure is defined as no achievement of CR after completion of initial treatment (including IFRT for certain patients). Secondary end points included the probability of overall survival (OS), disease-free survival (DFS), rate of CR after completion of initial treatment (including radiotherapy) and toxic effects (graded according to the NCI Common Terminology Criteria for Adverse Events, version 3). Responses including complete remission (CR), partial remission (PR), stable disease (SD) and progressive disease (PD) were assessed using the revised criteria of Cheson et al. ${ }^{[10]}$. OS and DFS were defined according to the NCI Workshop definitions ${ }^{[37]}$.

\section{Statistical analysis}

The probabilities of EFS, DFS and OS were estimated with the Kaplan-Meier method and were compared between groups with the use of the log-rank test. The rate of CR was compared between groups using a chi-square $(x 2)$ test. Univariate analysis was performed to assess the factors for short or long-term outcomes, including mediastinal disease, age, histology, ESR (A>50, B>30mm/h), B-symptoms, involved anatomic sites, extranodal disease, IPS, stage, and biochemical abnormalities (ALB, LDH, AKP, $\beta 2-\mathrm{MG}$ ). In multivariate analysis, the independent prognostic significance of variables for response was tested by logistic regression. Cox proportional hazards regression model was performed to identify independent prognostic significance of variables for EFS, OS and DFS. All p values were two-sided and $\mathrm{p}$ values less than 0.05 were considered statistically significant. All calculations were performed using the SPSS version 13.0.

\section{Results}

\section{Patient demographic and clinical features}

A total of 150 patients from 4 participating institutes were enrolled and analyzed within the study period. Males were affected more often than females (1.67:1). The median age was 30 y (range: 15-91 
y), the peak age of onset was 20-40 y involving 89 patients (59.3\%). No other peak at advanced age was noted, and only 12 patients were over $60 \mathrm{y}$ old. According to the updated 2008 WHO classification, nodular sclerosis was the most common histological subtype $(61.3 \%)$, followed by mixed cellularity (28.7\%). The most common symptom at diagnosis was lymphadenopathy (108cases, $72.0 \%$ ), of which the most frequent type was cervical lymphadenopathy (70.3\%). According to Ann-Arbor staging, 78 patients $(52.0 \%)$ had early-stage (I-II) HL, and the rest 72 patients (48.0\%) had advanced-stage (III-IV) HL. Imaging showed the presence of mediastinal mass in 88 patients; 16 of them had bulky mediastinal mass, as defined previously. In our study, 43 patients had extranodal sites, with the involvement of liver, lung, bone/bone marrow being the most common (69.7\%). Some other cases such as skin, chest wall, cartilage, breast and brain were noted as well. Their general clinical characteristics were shown in Table 1.

\section{Therapeutic outcome}

All 150 patients received initial chemotherapy. In addition to the aforementioned non-ABVD regimens for individual patients, the majority of patients were treated with 6 courses of ABVD regimen ( 9 additional patients with advanced-stage HL received 8 courses while 5 patients with early-stage HL received only 4 courses).Therapeutic modalities are summarized in Table1.Data on the interim and final response after chemotherapy or completion of all initial treatments (including RT) were available in 148 patients. At the end of chemotherapy, 98 patients achieved CR evaluated by CT or PET/CT. IFRT was recommended for patients with CR or PR after induction chemotherapy who had initial bulky mediastinal disease or areas of residual disease. Finally, 68 patients received IFRT. After the completion of initial treatment, the CR rate was $73.65 \%$, the overall response rate (ORR) was $83.11 \%$, 10 patients with a PR had an improvement to CR, and 4 patient with SD had an improvement to either CR (in 1 patient) or PR (in 3 patients). Another patient with SD showed progression after RT (Table 2).

At the end of study, 22 deaths were documented, as described below. With salvage treatment given to those with refractory or progressive diseases after initial treatment, a total of 119 patients achieved CR (including those with CR after initial therapy). Of these patients, 26 had a relapse during the follow-up after first remission. We documented an overall estimated 5-y EFS of 61.1\%, 5-y OS of $84.7 \%$, and 5-y DFS of $78.8 \%$.

\section{Prognostic factors}

\section{- Univariate analysis}

Table 3 shows the univariate analysis of risk factors associated with short or long-term outcomes. Namely, in our cohort, only 16 of the 88 patients with mediastinal mass had bulky mediastinal mass. Considering the sample size, we selected 'mediastinal mass' (involvement of mediastinal nodal) in univariate analysis. Regarding the primary end point, nodal site $\geqslant 3$; B-symptoms; extranodal disease; IPS (3-7); advanced stage; $\operatorname{ESR}(\mathrm{A}>50, \mathrm{~B}>30 \mathrm{~mm} / \mathrm{h}) ; \mathrm{ALB}<3.5 \mathrm{~g} / \mathrm{dl}$ and presence of two or more biochemical abnormalities(defined previously) were all identified as the significant unfavorable prognostic factors for 5-year EFS $(\mathrm{P}<0.05)$. Moreover, the first 5 variables also exerted adverse effect on 5-y OS, 5-y DFS and the rate of CR (P<0.05). The last 2 variables, except for their unfavorable impact on EFS, mainly favored a poor prognosis for the rate of $\mathrm{CR}(\mathrm{P}<0.01)$. ESR $(\mathrm{A}>50, \mathrm{~B}>30 \mathrm{~mm} / \mathrm{h})$ was also revealed as a poor prognostic factor for 5-year DFS and the rate of $\mathrm{CR}(\mathrm{P}<0.05)$, but not for 5-year OS $(\mathrm{P}=0.33)$. In addition, our univariate analysis failed to show a significant predictive value of age, mediastinal mass, histological type, serum LDH/AKP/ $\beta 2-\mathrm{MG} />1 \mathrm{xNormal}$ with respect to any 
outcome measure $(\mathrm{P}>0.05)$.

\section{- Multivariate analysis}

Multivariate analysis of covariates (Table 4) in the Cox and Logistic regression model demonstrated that the following remained as independent prognostic factors for the primary end point (EFS): B symptoms (HR=2.139, $\mathrm{p}=0.029,95 \%$ CI: 1.081-4.230), extranodal involvement (HR=2.208, $\mathrm{p}=0.034,95 \%$ CI: $1.054-3.903$ ), and IPS $\geq 3$ (HR=2.116, $\mathrm{p}=0.045,95 \%$ CI: $1.085-4.547)$. In addition, the rate of $\mathrm{CR}$ was independently affected by extranodal involvement $(\mathrm{OR}=5.724, \mathrm{p}=0.001,95 \% \mathrm{CI}$ : 1.964-16.683) and IPS $\geq 3(\mathrm{OR}=3.606, \mathrm{p}=0.047,95 \%$ CI: 1.017-12.779). Meanwhile, B symptoms was proved to be the unique independent adverse factor for DFS (HR=2.855, $\mathrm{p}=0.046,95 \% \mathrm{CI}$ : 1.018-8.005). Regarding the 5-year OS, no independent risk factors were observed.

\section{Stratification assessment}

\section{- Favorable and unfavorable group based on risk factors}

We divided patients into 2 groups according to the results of multivariate analysis: unfavorable group (with at least one of the following risk factors at initial diagnosis: B symptoms, extranodal involvement and IPS $\geqslant 3$ ) and favorable group (without any of these 3 factors). As shown in Figure 1, significant differences were detected in any outcome measures between patients categorized into the favorable $(\mathrm{n}=50,33.37 \%)$ and unfavorable cohorts $(\mathrm{n}=100,66.67 \%)$. Estimated 5-y EFS was $94.23 \%$ vs.43.20\%, p<0.0001; estimated 5-y OS was $96.67 \%$ vs.79.7\%, p=0.0021; estimated 5-y DFS was $96.43 \%$ vs. $64.60 \%, \mathrm{p}<0.0001$; and the rate of $\mathrm{CR}$ at completion of initial treatment was $98.0 \%$ vs. $61.2 \%, \mathrm{p}<0.001$. The positive predictive value (PPV) for EFS was $54 \%$, whereas the negative predictive value (NPV) was $96 \%$.

\section{- Interim imaging evaluation}

In our study, 67 of the 148 patients (45.27\%) with available data on interim evaluation underwent PET scan. Other 81 patients were evaluated by traditional CT. A total of 50 patients (33.8\%) achieved interim complete remission (CRi). As shown in Figure 2, patients who failed to reach CRi ( $\mathrm{n}=98$, 66.2\%) obtained significantly inferior EFS, DFS and CR at completion of initial treatment (5-y EFS: $45.7 \%$ vs. $89.6, \mathrm{P}<0.0001$; 5 -y DFS: $71.0 \%$ vs. $85.9 \%, \mathrm{P}=0.0406$; $\mathrm{CR}$ rate: $60.2 \%$ vs. $100 \%, \mathrm{p}<0.001$, respectively). No significant difference in the 5-year OS was indicated $(81.4 \%$ vs. $92.8 \%, \mathrm{P}=0.052)$. The PPV for EFS was $52 \%$, whereas the NPV was $94 \%$.

\section{- A new risk model}

To further assess the added value of traditional risk factors and interim imaging evaluation, we establish a new risk model combining the interim response with favorable/unfavorable group as defined above. Three risk groups with significantly different outcomes were thus identified: favorable group with $\mathrm{CRi}(\mathrm{n}=27,18.2 \%)$, unfavorable group or non-CRi $(\mathrm{n}=46,31.1 \%)$, and unfavorable group with non-CRi $(\mathrm{n}=75,50.7 \%)$. The estimated 5-y EFS in these 3 subset were $100 \%, 83.1 \%$ and $33.1 \%$, respectively $(\mathrm{P}<0.0001)$. Similarly, the 5 -y OS were $100 \%, 89.4 \%$ and $77.6 \%$, respectively $(\mathrm{P}=0.0063)$. The 5-y DFS were 100\%, 80.98\% and 57.0\%, respectively ( $\mathrm{P}=0.0002)$, as shown in Figure 3. The rate of $\mathrm{CR}$ at completion of initial treatment in these 3 subsets were $100 \%, 97.8 \%$ and $49.3 \%$, respectively $(\mathrm{P}<0.001)$. The PPV for EFS was $65.3 \%$, whereas the NPV was $90.4 \%$. When the intermediate risk 
group was further refined into 2 subgroups: favorable group without CRi and unfavorable group with CRi. 5-y EFS, OS, and DFS were slightly higher in the former than in the latter (88.3\% vs. $77.3 \%$; $92.9 \%$ vs. $85.6 \%$; $92.3 \%$ vs. $70.2 \%$, respectively), despite no significant difference was indicated $(\mathrm{P}>0.05)$.

\section{Adverse events}

Neutropenia was identified as the most common hematologic toxicity during the treatment period. Overall, 59 patients (39.33\%) developed grade 3 or 4 neutropenia. In addition to fever of unknown origin, documented infectious episodes occurred in 29 patients: pneumonia (17); sepsis (5); herpes (3); skin infection (2); and gastroenteritis (2). Regarding the non-hematologic toxicity, 8 patients experienced grade 3 or 4 hepatic toxic effects during chemotherapy; in all cases, the toxic effects were resolved with temporary discontinuation of chemotherapy and administration of specific drugs. Moreover, 3 patients with no history of cardiopathy appeared cardiotoxicity after chemotherapy by ABVD regimen. Other 2 patients had interstitial pneumonia (one of them developed cytomegalovirus retinitis caused by high-dose corticosteroids). Radiation pneumonitis after IFRT to mediastinal mass was reported in 2 cases. In our study, only 1 patient developed secondary neoplasia (lung carcinoma) 7 $\mathrm{y}$ after chemotherapy and eventually died of this disease; this low number is attributed to a relatively short follow-up (median follow-up of 48.5 months). Except for this case of death, 21 additional deaths were recorded, 11 of which were treatment-related (6 cases of death from pneumonia, 2 from severe sepsis, 1 from cardiovascular event, 1 from radiation pneumonitis, and 1 from pulmonary fibrosis). The other deaths resulted from initial disease progression.

\section{Discussion}

Studies have consistently found a relatively low incidence of HL in China. Based on our study, the general clinical features are rather comparable to Caucasian patients ${ }^{[14]}$.As reported in a large number of studies, HL occurs more frequently in males than females ${ }^{[12,13]}$. The percentage of males were $59.4 \%$ and $53.3 \%$ as shown in GHSG HD13 and HD18 studies ${ }^{[13]}$. Out study showed that the ratio of male to female was $1.67: 1$, which was slightly higher than that of Western patients; however, no significant difference was indicated. Regarding to the histopathological subtypes, the distribution in our patients was similar to that of the Western pattern. The nodular sclerosis is the most common subtype followed by mixed cellularity ${ }^{[15,16]}$. However, our data showed slight characteristic difference. A dual peak age distribution was not observed in our cohort; just an early peak appeared in young adults, which could be attributed to the relatively small sample size or ethnic differences.

With recent advances in radiation therapy and combination chemotherapy regimens, the cure rate of patients with HL has been significantly increased ${ }^{[20]}$. Reports in Western countries accumulated data for patients with early-stage HL. In brief, CR rate ranged from 92\% to 96\%; 5-y OS ranged from 94 to $97 \%$ and 5-y FFTF ranged from $85 \%$ to $92 \%{ }^{[17,18]}$. Regarding to the patients with advanced-stage HL, their CR rate was from $65 \%$ to $76 \%$; 5-y OS was from $80 \%$ to $90 \%$ and 5-y FFTF was from $60 \%$ to $65 \%{ }^{[5,19]}$. Compared with previous data, the short or long-term outcome of our patients were slightly inferior to those of Western patients. The difference may be attributed to the factors listed below.

(1) Geographic and ethnic factors: According to a report from June-Won Cheong et al. ${ }^{[14]}$, the findings for $105 \mathrm{HL}$ patients (regardless of the disease stage) were as follows: 5-year DFS, 79.2\%; 5-year OS, $84.8 \%$; and ORR, $87.6 \%$. Similar results were also reported in a retrospective study 
involving 67 Chinese patients in Hong Kong ${ }^{[21]}$, which showed a 5-year OS of $89 \%$ for all patients, $74.7 \%$ for patients with advanced-stage HL and $95 \%$ for patients with early-stage HL. All of these figures were lower than those reported for the Caucasian patients, but similar to the results obtained in our study.

(2) Insufficient combined chemo-radiotherapy: NCCN and many large international study groups recommended that for patients with early-stage HL, chemotherapy with IFRT is currently recognized as the standard therapy. For patients with advanced stage, radiotherapy remains controversial. In accordance with this principle, excluding a patient with early-stage HL who progressed during induction therapy and switched for salvage treatment, at least 77 patients with early-stage HL in our cohort should have received chemo-radiotherapy ideally. Nevertheless, in our study, IFRT was conservatively recommended to patients in any stages with CR or PR after induction chemotherapy who had initial bulky mediastinal disease or areas of residual disease. Finally, of the 68 patients who underwent IFRT, 40 had early-stage HL, indicating that at least $37 / 77(48.1 \%$ ) of patients with early-stage HL should have received IFRT but did not. Furthermore, we compared outcomes stratified by different treatment modalities (chemotherapy alone vs combined chemo-radiotherapy). Patients with combined chemo-radiotherapy showed a tendency to obtain higher EFS, OS and DFS than patients with chemotherapy alone (5-y EFS: $62.8 \%$ vs 57.3\%; 5-y DFS: $82.3 \%$ vs $71.7 \%$; 5-y OS: $86.8 \%$ vs $84.3 \%$, p>0.05, respectively), despite no significant difference was detected. This finding might explain for the inferior therapeutic outcomes in our present study due to insufficient combined chemo-radiotherapy.

In our study, the majority of traditional risk factors that have been widely accepted ${ }^{[3,26]}$ remained prognostic impact with respect to CR rate, EFS, DFS or OS in the univariate analysis. However, bulky mediastinal mass whose prognostic value has been identified by large study groups such as EORTC and GHSG ${ }^{[27,29]}$ failed to be verified in our cohort. On the basis of our data, only 16/88 cases of mediastinal mass could be defined as bulky mediastinal mass. Patients with bulky mediastinal mass showed a slightly worse outcome, and no significant difference was indicated. Estimated 5-year EFS and OS was $61.11 \%$ vs. $62.38 \%$ and $82.96 \%$ vs. $85.57 \%$, p>0.05, respectively. We proposed the following hypotheses: First, this finding might be due to a relatively short follow-up or limited sample size. Second, the measurement of bulk is limited to single large mass. It could underestimate the total tumor burden in patients with diffuse disease. For this reason, the presence of bulky mediastinal mass was always not a risk factors for advanced stage $\mathrm{HL}^{[3]}$. This could probably explain for no significant influence of bulky mediastinal mass among all patients regardless of stages in our study. With sample size considered, we selected 'mediastinal mass' in univariate analysis irrespective of whether it was bulky mediastinal disease or not. Similarly, no significant difference was found (as shown in Table 3), for which one of the reasons might be attributed to the relatively small proportion of bulky mediastinal mass. In addition, various factors related to tumor included the number of tumor cells, growth characteristics, etc. Bulky mediastinal disease describes the large number of tumor cells as well as rapid growth. This would be more representative and convincing than simple involvement of mediastinal nodal as a variable.

Interim PET/CT was recently proposed by a number of researchers as a new tool to predict therapy outcome. It shows excellent outcomes for early PET-patients and rather poor outcomes for early PET+ patients ${ }^{[7,30-32]}$. In patients with advanced disease, the high prognostic value of early FDG-PET even over shadowed the role of IPS ${ }^{[33,34]}$. Consistent with previous reports, in our cohort, of 67 patients who underwent interim PET scan, 39 (58.2\%) showed a negative interim PET (iPET-), 
whereas the remaining 28 patients showed a positive interim PET (iPET+). Comparison between patients with iPET- and iPET+ indicated a significantly different outcome (5y-EFS: 86.7\% vs. 37.2\%, $\mathrm{P}<0.001)$. However, owing to health insurance issue, one should note that PET/CT is not widely used in China, as well as in many other developing countries. Thus, patients in our present study received interim evaluation both by traditional CT or PET/CT, which also revealed a significant influence with respect to EFS, DFS and CR.

In a simplified way, prognostic factors of tumors can be classified into disease-related factors, host-related factors, and treatment-related factors. As in the retrospective study by Chow et al. ${ }^{[35]}$ involving 76 DLBCL patients, the prognostic accuracy for PFS of PET result was limited(C=0.63), as it was for IPI $(\mathrm{C}=0.75)$. However, with the two indicators combined, the predictive accuracy was improved $(\mathrm{C}=0.81)$. A similar study by Lanic $\mathrm{H}$ et al. ${ }^{[36]}$ showed that a score incorporating aaIPI, interim response and $\mathrm{GCB} / \mathrm{ABC}$ classification divided patients into 2 groups with significantly distinct outcomes. In the present study, IPS, B symptoms and extranodal involvement (3 independent prognostic factors in multivariate analysis) reflected pretreatment factors related to tumor burden and host, whereas the interim-response was related to chemosensitivity. Prompted by previous studies, we proposed a new risk model that combined covariates from the baseline and the ones during treatment. It would be more adaptable in modern treatment, and probably compensate the limitation related to imaging technique, particularly in developing countries. Three final risk groups were thus established. Eventually, the EFS, DFS and OS curves among risk groups derived from our final model as well as the CR rate showed significant differences. The favorable group combined with CRi identified a subset of patients with a low risk of failure, reaching 5-y EFS at $100 \%$. Conversely, unfavorable group combined with non-CRi is related to a high risk of treatment failure, reaching $66.9 \%$ at 5 years. This finding indicated that the combination of traditional risk factors and the interim evaluation could be a new tool to predict prognosis. Namely, with the comprehensive comparison of PPV and NPV for EFS among the new risk model, interim evaluation and favorable/unfavorable group, the new risk model shows potential as a more powerful predictive tool.

In conclusion, our study is slightly limited by its retrospective nature and relatively small sample size; however, it can provide additional information on HL in the Asian population. Generally, the overall clinical features are comparable to those of Caucasian patients, whereas the treatment outcomes are slightly worse than those reported for western patients. B symptoms, extranodal disease and IPS are the independent prognostic factors with respect to EFS. With the advances in radiological imaging, traditional risk factors still remain their certain impact on prognosis. Moreover, the new risk model in our study combining traditional factors and interim evaluation by PET/CT or CT is regarded as a more powerful tool in risk assessment, particularly for patients in developing countries. The model can be used to propose a better risk-adapted therapeutic strategy, which requires further validation in an independent patient population.

\section{Acknowledgements}

This work was supported in part by the International Cooperation Projects of Shanghai Science and Technology Committee (15410710200), Shanghai Leading Talent Projects (2016008), Academic Leader Program of Shanghai Science and Technology Committee (16XD1402000)

\section{Conflicts of interest}

None declared. 


\section{References}

[1] Siegel R, Naishadham D, Jemal A. Cancer statistics, 2012. CA Cancer J Clin. 2012; 62(1):10-29.

[2] Eich HT, DiehlV, Görgen H, Pabest T,Markova J,Debus J et al. Intensified chemotherapy and dose reduced involved-field radiotherapy in patients with early unfavorable Hodgkin's lymphoma: final analysis of the German Hodgkin Study Group HD11 trial. Journal of Clinical Oncology.2010; 28(27): 4199-206.

[3] Hasenclever D, Diehl V. For the International Prognostic Factors Project on advanced Hodgkin's disease. A prognostic score for advanced Hodgkin's disease. N Engl J Med.1998; 339(21):1506-1514.

[4] Bonfante V, Santoro A, Viviani S, Valagussa P, Bonadonna G. ABVD in the treatment of Hodgkin Lymphoma. Semin Oncol. 1992; 19: 38-44.

[5] Hoskin PJ, Lowry L, Horwich A, Jack A, Mead B, Hancock BW et al. Randomized comparison of the Stanford V regimen and ABVD in the treatment of advanced Hodgkin's lymphoma: United Kingdom National Cancer Research Institute Lymphoma Group Study ISRCTN 64141244. J Clin Oncol. 2009; 27 (32):5390-6.

[6] Viviani S, Zinzani PL, Rambaldi A, Brusamolino E, Levis A, Bonfante V et al.ABVD versus BEACOPP for Hodgkin's lymphoma when highdose salvage is planned. N Engl J Med. 2011; 365(3):203-12.

[7] Hutchings M, Loft A, Hansen M, Pedersen LM, Buhl T, Jurlander J et al. FDG-PET after two cycles of chemotherapy predicts treatment failure and progression-free survival in Hodgkin lymphoma. Blood. 2006; 107(1): 52-59.

[8] Rossi C, Kanoun S, Berriolo-Riedinger A, Dygai-Cochet I, Humbert O, Legouge C et al. Interim 18F-FDG PET SUVmax Reduction Is Superior to Visual Analysis in Predicting Outcome Early in Hodgkin Lymphoma Patients. J Nucl Med. 2014; 55(4):569-73.

[9] Lister TA, Crowther D, Sutcliffe SB, Glatstein E, Canellos GP, Young RC et al. Report of a committee convened to discuss the evaluation and staging of patients with Hodgkin's disease: Cotswolds meeting. J Clin Oncol. 1989; 7(11):1630-1636.

[10] Cheson BD, Pfistner B, Juweid ME, Gascoyne RD, Specht L,Horning SJ et al. International Harmonization Project on Lymphoma. Revised response criteria for malignant lymphoma. J Clin Oncol. 2007; 25(5):579-86.

[11] Swerdlow S, Campo E, Harris N, Jaffe E, Pileri S, Stein H. WHO classification of tumours, vol. 2. Lyon: International Agency for Research on Cancer 2008.

[12] Schwenkglenks M, Pettengell R, Szucs TD, Culakova E, Lyman GH. Hodgkin lymphoma treatment with ABVD in the US and the EU: neutropenia occurrence and impaired chemotherapy delivery. J Hematol Oncol. 2010; 3:27.

[13] Sauer M, Plütschow A,Jachimowicz RD, Kleefisch D, Reiners KS, Ponader S et al. Baseline serum TARC levels predict therapy outcome in patients with Hodgkin lymphoma. Am J Hematol. 2013; 88(2):113-5.

[14] Cheong JW, Park SY, Roh JK, Suh CO, Hahn JS. Treatment of Hodgkin's Disease: A Twenty-Year Follow-up of Patients at a Center in Korea. Yonsei Medical Journal. 2006; 47(4):455-465.

[15] Evens AM, Altman JK, Mittal BB, Hou N, Rademaker A, Patton D et al. Phase I/II trial of total 
lymphoid irradiation and high-dose chemotherapy with autologous stem-cell transplantation for relapsed and refractory Hodgkin's lymphoma. Annals of Oncology. 2007; 18(4): 679-688.

[16] Brusamolino E, Baio A, Orlandi E, Arcaini L, Passamonti F, Griva V et al. Long-term Events in Adult Patients with Clinical Stage IA-IIA Nonbulky Hodgkin's Lymphoma Treated with Four Cycles of Doxorubicin, Bleomycin, Vinblastine, and Dacarbazine and Adjuvant Radiotherapy: A Single-Institution 15-Year Follow-up. Clin Cancer Res. 2006; 12(21): 6487- 6493.

[17] Engert A, Plütschow A, Eich HT, Lohri A, Dörken B, Borchmann P et al. Reduced treatment intensity in patients with early-stage Hodgkin'slymphoma. N Engl J Med. 2010; 363(7): 640-652.

[18] Eich HT, Diehl V, Görgen H, Pabst T, Markova J, Debus J et al. Intensified chemotherapy and dose reduced involved-field radiotherapy in patients with early unfavorable Hodgkin's lymphoma: Final analysis of the German Hodgkin Study Group HD11 trial. J Clin Oncol. 2010; 28(27):4199-4206.

[19] Duggan DB, Petroni GR, Johnson JL,Glick JH, Fisher RI, Connors JM et al. Randomized comparison of ABVD and MOPP/ABV hybrid for the treatment of advanced Hodgkin's disease: Report of an intergroup trial. J Clin Oncol. 2003; 21(4):607-614.

[20] Marri PR, Ansell SM. Progress in the initial management of Hodgkin's Lymphoma. Transfus Apher Sci. 2013; 49(1):12-8.

[21] Law MF, Ng TY, Chan HN, Lai HK, Ha CY, Leung C et al. Clinical features and treatment outcomes of Hodgkin's lymphoma in Hong Kong Chinese. Arch Med Sci. 2014; 10(3):498-504.

[22] Garcia R, Hernández JM, Caballero MD, González M, Galende J, del Cañizo MC et al. Serum lactate dehydrogenase level as a prognostic factor in Hodgkin's disease. Br J Cancer. 1993; 68(6):1227-31.

[23] Itoh K, Kinoshita T,Watanabe T, Yoshimura K, Okamoto R, Chou T et al.Prognostic analysis and a new risk model for Hodgkin lymphoma in Japan. Int J Hematol. 2010; 91(3):446-55.

[24] Chronowski GM, Wilder RB, Tucker SL, Ha CS, Sarris AH, Hagemeister FB et al. An elevated serum b-2-microglobulin level is an adverse prognostic factor for overall survival in patients with early-stage Hodgkin disease. Cancer. 2002; 95(12):2534-8.

[25] Vassilakopoulos TP, Nadali G, Angelopoulou MK, Siakantaris MP, Dimopoulou MN, Kontopidou FN et al. The prognostic significance of b2-microglobulin in patients with Hodgkin's lymphoma. Haematologica. 2002; 87(7):701-8.

[26] Engert A, Franklin J, Eich HT, Brillant C, Sehlen S, Cartoni C et al. Two cycles of doxorubicin, bleomycin, vinblastine, and dacarbazine plus extended-field radiotherapy is superior to radiotherapy alone in early favorable Hodgkin's lymphoma: final results of the GHSG HD7 trial. J Clin Oncol. 2007; 25(23):3495-502.

[27] Eichenauer DA, Engert A, Dreyling M, ESMO Guidelines Working Group. Hodgkin's lymphoma: ESMO Clinical Practice Guidelines for diagnosis, treatment and follow-up. Ann Oncol. 2011; 22 Suppl 6:vi55-8.

[28] Bröckelmann PJ, Angelopoulou MK, Vassilakopoulos TP. Prognostic factors in hodgkin lymphoma. Semin Hematol. 2016; 53(3):155-64

[29] Klimm B, Goergen H, Fuchs M, von Tresckow B, Böll B, Meissner J et al. Impact of risk factors on outcomes in early-stage Hodgkin's lymphoma: an analysis of international staging definitions. Ann Oncol.2013; 24(12):3070-6.

[30] Hutchings M, Mikhaeel NG, Fields PA, Nunan T, Timothy AR. Prognostic value of interim FDG-PET after two or three cycles of chemotherapy in Hodgkin lymphoma. Ann Oncol. 2005; 
16(7): 1160-1168.

[31] Zanzani PL,Rigacci L, Stefoni V, Broccoli A, Puccini B, Castagnoli A et al. Early interim 18F-FDG PET in Hodgkin's lymphoma: evaluation on 304 patients. Eur J Nucl Med Mol Imaging. 2012; 39(1):4-12.

[32] Cerci JJ, Pracchia LF, Linardi CC, Pitella FA, Delbeke D, Izaki M et al. 18F-FDG PET After 2 Cycles of ABVD Predicts Event-Free Survival in Early and Advanced Hodgkin Lymphoma. J Nucl Med. 2010; 51(9):1337-43.

[33] Gallamini A, Rigacci L, Merli F, Nassi L, Bosi A, Capodanno I et al. The predictive value of positron emission tomography scanning performed after two courses of standard therapy on treatment outcome in advanced stage Hodgkin's disease. Haematologica. 2006; 91(4):475-481.

[34] Gallamini A, Hutchings M, Rigacci L, Specht L, Merli F, Hansen M et al.Early interim2-[18F] fluoro-2-deoxy-D-glucose positron emission tomography is prognostic ally superior to international prognostic score in advanced-stage Hodgkin's lymphoma: a report from a joint Italian-Danish study. J Clin Oncol. 2007; 25(24):3746-3752.

[35] Chow A, Phillips M, Siew T, Cull G, Augustson B, Ward M et al. Prognostic nomogram for diffuse large B-cell lymphoma incorporating the International Prognostic Index with interim-positron emission tomography findings. Intern Med J. 2013; 43(8):932-9.

[36] Lanic H, Mareschal S, Mechken F, Picquenot JM, Cornic M, Maingonnat C et al. Interim positron emission tomography scan associated with international prognostic index and germinal center B cell-like signature as prognostic index in diff use large B-cell lymphoma. Leuk Lymphoma. 2012; 53(1):34-42.

[37] Cheson BD, Bennett JM, Kopecky KJ et al. Revised recommendations of the International Working Group for Diagnosis, Standardization of Response Criteria, Treatment Outcomes, and Reporting Standards for Therapeutic Trials in Acute Myeloid Leukemia. J Clin Oncol 2003;21: 4642-9.

\section{Legends}

Table 1. Demographics and baseline characteristics

Table 2. Responses at completion of chemotherapy and completion of all initial treatment (including RT)

Table 3. Univariate analysis of risk factors

Table 4. Multivariate analysis of risk factors

Figure 1: Kaplan-Meier curves of EFS, OS and DFS for patients according to the favorable or unfavorable group. (a) Curves of Event-free survival. (b) Curves of Overall survival. (c) Curves of Disease-free survival.

Figure 2: Kaplan-Meier curves of EFS, OS and DFS for patients according to the interim imaging evaluation. (a) Curves of Event-free survival. (b) Curves of Overall survival. (c) Curves of Disease-free survival.

Figure 3: Kaplan-Meier curves of EFS, OS and DFS for patients according to the new risk group. (a) Curves of Event-free survival. (b) Curves of Overall survival. (c) Curves of Disease-free survival 